\title{
Survey on Classification of Clustering Technique
}

\author{
Varchika Gupta ${ }^{1}$, Ashutosh Rastogi ${ }^{2}$
}

M.Tech Scholar, Wireless Communication and Sensor Network, Department of Electronics and Communication,

$$
\text { BBD University }{ }^{1,2}
$$

\begin{abstract}
Wireless Sensor Networks (WSNs) in the zone time alter to more engaging and broaden their way into immense variance of exercising in different domains. Elevation in wireless sensor network technologies provide the affability of small and low-cost sensor nodes with the adequacy of sensing, processing, computing the physical and environment circumstances. Due to inhibitionin transmission range, processing and energy resources it is requisite to design impressive and energy aware protocol to enhance network lifetime.Routing techniques are develop to increase life cycle of sensor network and enhance throughput performance. This paper gives, an analysis of different routing strategies used in wireless sensor networks further importance of cluster based routing, types of cluster, classification of cluster based routing are discussed. The study concludes with the issues and the resent researchers on cluster based routing.
\end{abstract}

Keywords: Wireless Sensor Network, Routing Schemes, Clustering-based Routing.

\section{INTRODUCTION}

Now a day's wireless sensor networks are mostly used in civilian application areas such as monitoring, tracking, traffic control and health applications. WSN consist of wireless modules called sensor nodes [1]. Architecture of sensor nodes is as follows:

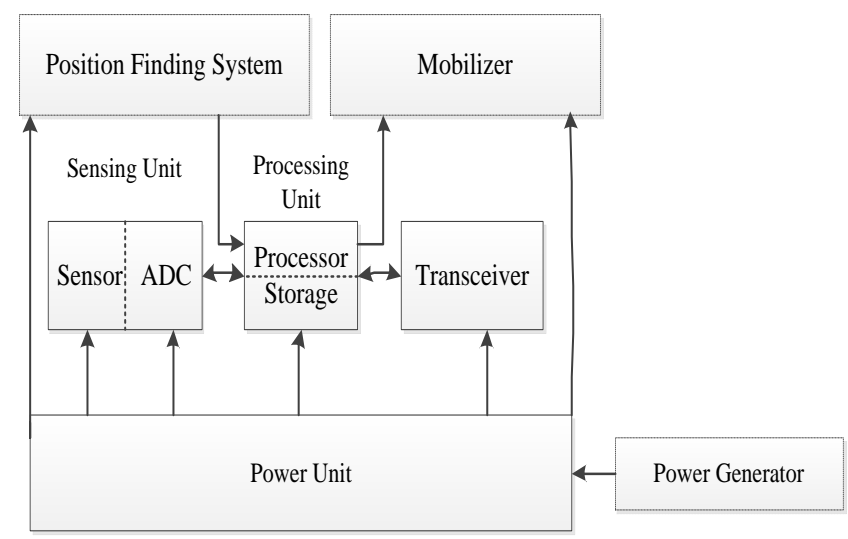

Fig.1 Components of sensor nodes

Due to limitation on transmission bandwidth, power supply and processing capability, efficient routing become important in wireless sensor network. According to the network structure routing in WSN is divided into two types: flat routing and hierarchical routing. In flat routing, all the node preform the same task and have same functionality in network. Generally Flooding technique are used in which information dissemination is done hop by hop.It is most effective in small-scale networks [2].

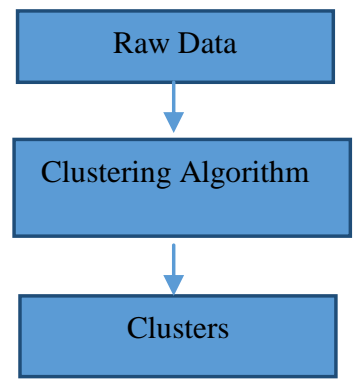

Fig. 2 Stages of Clustering 
They become undesirable for the large scale networks due to limited resources and more requirement of bandwidth and data processing. In hierarchical routing all node have to execute different tasks and they mostly grouped into clusters on the basis of requirements.[3] It is task of making grouping set of object in group that must be similar to one another and different from the objects in other groups.Clustering is useful when similarity and difference between the groups are greater.

Grouped cluster have nodes with different energies in which higher energy nodes are consider as the cluster heads and they perform the task of data processing and information dissemination, while nodes with lower energy acts as member nodes and preform information sensing.[5] Each node of the cluster directly communicate with cluster head, then cluster head forward the sensed data to Base station (BS). Cluster heads can communicate with each other in the network or directly to base station.

Clustering algorithm has variety of advantages such as scalability, less load, less energy consumption and more robust. Clustering of nodes minimizes residual energy and maximize network performance.[6] This improves the overall network traffic and reduces network lifetime.

\section{CLASSIFICATION OF WSN ROUTING PROTOCOLS}

On the basis of network structure, WSN routing protocols are classified into Flat based, hierarchical (cluster) based and location based routing protocols.

Flat Based Routing

In this all nodes are equal and act same functionality. Due to the dense deployment and dynamic environment it is not possible to assign global identifiers to each node of wireless sensor networks.

Location Based Routing

Most routing protocols require location information for sensor nodes in wireless sensor networks. To calculate distance between two nodes location information is necessary to estimate energy consumption on the basis of signal strength.

\section{Hierarchical Based Routing}

Scalability is consider as one of the essential design features sensor networks. Due to single gateway architecture gateway cause overload which cause break in communication and for tracking of events and also faces the problem in long distance communication.[5]

Hierarchical (cluster) based routing has been overcome the drawback with the advantages of scalability and efficient communication. [3]. It also maintain the energy consumption of sensor nodes and performs the data aggregation which reduces the problem of congestion occurs due to reduction in message transmission between the nodes and base station. Clustering routing is energy-efficient protocol that can be used by the sensor node to report sensed data to sink. In clustering, wireless sensor network are divided into several cluster of sensors. The communication in cluster is coordinated by the cluster head, which aggregates the data from each cluster of the network and transmit to the base station.

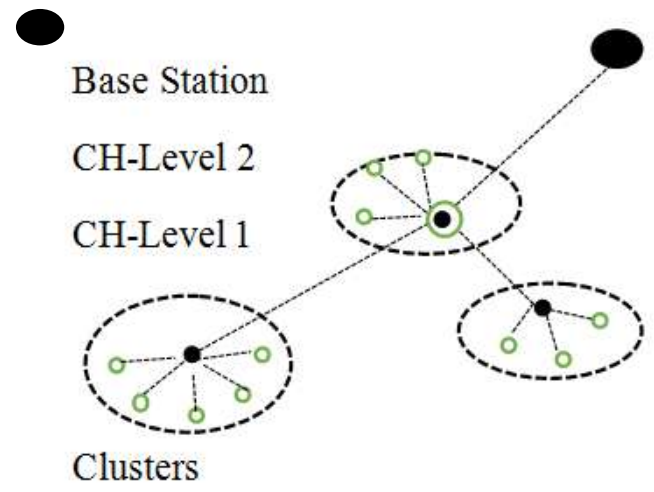

Fig.3 Cluster-based Hierarchical Model

As shown in figure 3, in hierarchical routing data are travels from lower clustered layer to higher one. Data also hops from one node to another, but when it hop from one cluster layer to another it covers larger distances. Hence the data reach faster to the base station. Clustering helps in inherent optimization capabilities at the cluster head. So, Clustering routing is best option for the network scalability and efficient communication.

Clustering algorithm are categorized into various types such as partitional, hierarchical, density and grid-based clustering. 


\section{GENERAL TYPES OF CLUSTER}

I. Well-separated cluster

Cluster is a collection of points so that any point in the cluster is nearest (that is more similar) to other points in the cluster as compared to any other point in that point is not in the cluster.

II. Contiguous cluster

A cluster is collection of points in which a point in cluster is nearest (more similar) to one or more other points in the cluster as compared to any point.

\section{Center-based cluster}

A cluster is collection objects in which the object in a cluster is nearest to the center (centroid) of a cluster, then to the center of any other cluster.

IV. Density-based cluster

Cluster points of the dense region which is separated from the region of high dense as a noise that is low dense region.

V. Shared property or conceptual cluster

Cluster that finds the common property or represents a particular.

\section{CLUSTER BASED ROUTING PROTOCOLS IN WSN}

When efficient communication and network scalability is required cluster routing is considered as energy efficient method. In which higher energy nodes are performs processing and sending data while lower energy nodes are used for sensing and sending information to cluster heads (CHs). Cluster based routing protocols are classified as block clusterbased, grid cluster based and chain cluster based routing.

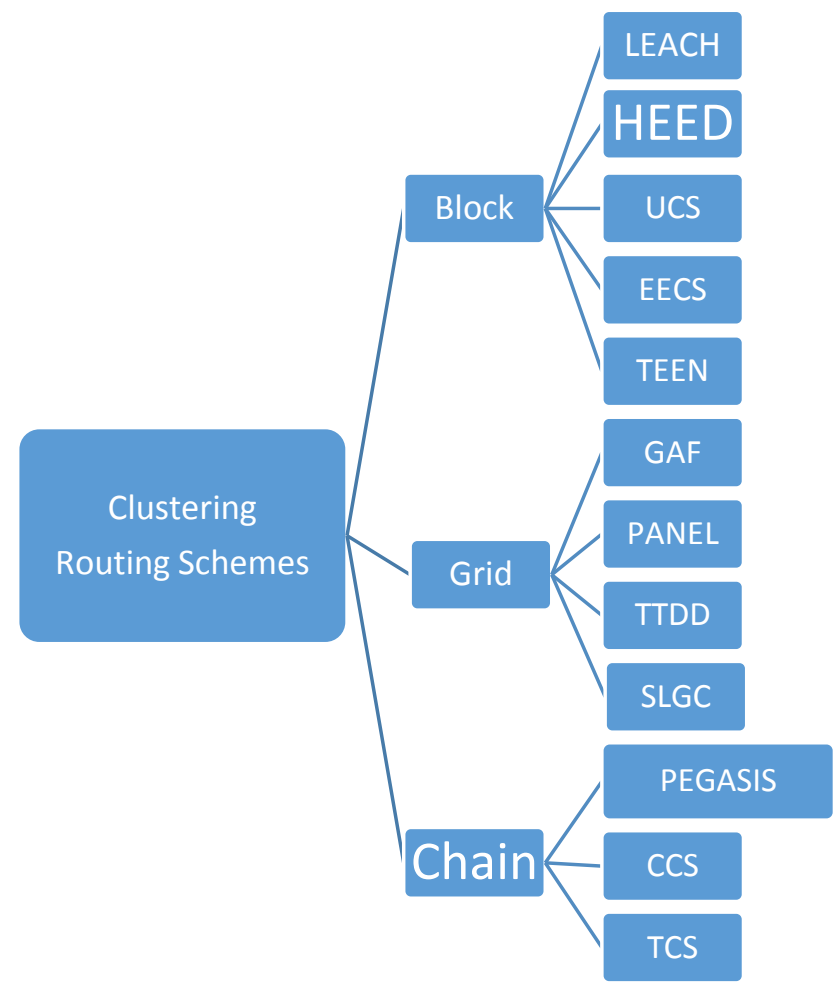

Fig.4 . Cluster-based routing schemes

\section{BLOCK CLUSTER-BASED ROUTING SCHEMES}

Block based Clustering: It is the efficient method for clustering used for the real-time detection.

LEACH Low-Energy Adaptive Clustering Hierarchy (LEACH) was the first Cluster based routing and it was proposed by Heinzelman. 


\section{IJARCCE}

The clustering operation in LEACH is done into two stages: setup phase and steady state phase. As during the setup phase cluster are organised and during the steady state phase data transmission takes place.

During communication with base station high energy dissipate, in LEACH the selection of node as a cluster head is done through rotation.[8] Then these cluster-head node broadcasts their data to other sensor node in the network. The sensor node of the network belongs to that cluster which requires minimum communication energy.

After complete cluster formation, cluster head aggregates the data and then transmit to the base station.

Table 1. Merit and Limitations of LEACH

\begin{tabular}{|l|l|}
\hline Merit & Limitation \\
\hline $\begin{array}{l}\text { As load is shared between the node and each } \\
\text { node have equal chance to become cluster } \\
\text { head in the subsequent round. }\end{array}$ & $\begin{array}{l}\text { Single hop communication takes place so LEACH } \\
\text { cannot be used for large scale networks. } \\
\text { It cannot provide the load balancing and cluster } \\
\text { To prevent congestion TDMA is used } \\
\text { hetween the cluster head selection. }\end{array}$ \\
\begin{tabular}{l} 
which uniform distribution cannot achieved \\
\hline
\end{tabular}
\end{tabular}

\section{HEED}

Hybrid Energy-Efficient distributed (HEED) was proposed by Younis and Fahmy.

In this cluster head selection is not random. Cluster are formed on the basics of the residual energy of node and intracluster communication cost.

Table .2. Merit and Limitations of HEED

\begin{tabular}{|l|l|}
\hline Merit & Limitation \\
\hline $\begin{array}{l}\text { Fully distributed cluster based routing. } \\
\begin{array}{l}\text { Achieves load balancing and uniform CH } \\
\text { distribution. }\end{array}\end{array}$ & $\begin{array}{l}\text { Unbalance energy consumption due to more number } \\
\text { of cluster. } \\
\text { Migh energy efficiency and scalable due to multi hop } \\
\text { communication. }\end{array}$ \\
$\begin{array}{l}\text { Several iterations are done to form cluster leads to } \\
\text { additional overhead. }\end{array}$ \\
\hline
\end{tabular}

\section{UCS}

Unequal Clustering Size (UCS) proposed by Soro et al.

Equal size clusters causes an unbalance load on the cluster heads, whereas unequal size cluster provide balance energy consumption for cluster heads.

Total number of nodes in the network depend on the residual energy of the cluster head. Each cluster head transmits it data to the closest cluster head in the direction of the base station, in this data is transmitted through multiple hops.

Table 3.Merit and Limitation of UCS

\begin{tabular}{|l|l|}
\hline Merit & Limitation \\
\hline $\begin{array}{l}\text { To maintain uniform energy consumption, the } \\
\text { number of nodes varies. } \\
\text { Low energy consumption as compared to LEACH. }\end{array}$ & $\begin{array}{l}\text { Cluster heads are predetermined as well as network } \\
\text { is not homogeneous. } \\
\text { It is not suitable for large range network as residual } \\
\text { energy is not consider. }\end{array}$ \\
\hline
\end{tabular}

\section{EECS}

Energy Efficient Clustering Scheme (EECS) was proposed by Ye et al.

In this the node which have more residual energy is elected as the cluster head. [7]. Across the network the number of cluster is uniform and divide the network into clusters and it uses direct communication between the cluster head and base station.

Table 4.Merit and Limitation of EECS

\begin{tabular}{|l|lll|}
\hline Merit & Limitation & & \\
\hline $\begin{array}{l}\text { Energy consumption and communication are balance in } \\
\text { the network. }\end{array}$ & $\begin{array}{l}\text { Congestion due to global information for } \\
\text { communication. } \\
\text { Large distance communication with base station requires } \\
\text { lot of energy for this dynamic sizing of clusters are used. }\end{array}$ & $\begin{array}{l}\text { Lof energy consume due to single hop } \\
\text { communication. }\end{array}$ & \\
\hline
\end{tabular}




\section{TEEN}

(TEEN) proposed by Anjeshwar et al.

It is combination of hierarchical and data-centric approach. It is mostly name as two-tier clustering topology and uses two threshold: hard threshold (HT) and soft threshold (ST).

Hard threshold is used for sensed attribute and soft threshold is used to show the small change in the sensed attribute. These data reduce data communication.

Table 5.Merit and Limitation of TEEN

\begin{tabular}{|l|l|}
\hline Merit & Limitation \\
\hline $\begin{array}{l}\text { Transmission of data are controlled by two } \\
\text { threshold values. }\end{array}$ & $\begin{array}{l}\text { When the values of the threshold are different, node } \\
\text { will not able to communicate. } \\
\text { If node will not able to communicate then data may } \\
\text { be lost. }\end{array}$ \\
\hline
\end{tabular}

\section{GRID CLUSTER-BASED ROUTING ALOGRITHMS}

Grid Cluster-based routing algorithm: The data space is quantized into finite number of cells in the form of grid and all the clustering operation are performed on grid.

\section{GAF}

Geographic Adaptive Fidelity (GAF) was proposed by Xu et al. and is this location-based routing algorithm is designed for mobile ad hoc networks.

The cluster in the GAF are virtual fixed grid, these virtual grids are small enough so that every node in cell can directly communicate with node[11]. In GAF a node have three states: (i) discover, to find neighbour in grid, (ii)active, reflecting participation in routing and (iii) sleep, when radio switched off.

Table 6. Merit and Limitation of GAF

\begin{tabular}{|l|l|}
\hline Merit & Limitation \\
\hline $\begin{array}{l}\text { Network lifetime is increase by saving energy. } \\
\text { Fidelity of routing is maintained. }\end{array}$ & Large traffic injection and delay is not predictable. \\
\hline
\end{tabular}

\section{PANEL}

Position-based Aggregator Node Election scheme (PANEL) proposed by Buttyan and Schaffer. PANEL uses position information of the node, to elect node as a node aggregator.

In PANEL, the nodes are positioned in bounded area partitioned into geographic clusters. The clustering operation is determined before network is deployed, and each node pre-loaded with geographic information of its cluster.

Table 7. Merit and Limitation of PANEL

\begin{tabular}{|l|l|}
\hline Merit & Limitation \\
\hline Energy efficient scheme ensures load balancing and & $\begin{array}{l}\text { Clusters are predetermined. } \\
\text { long network lifetime. }\end{array}$ \\
Supports Asynchronous applications. & $\begin{array}{l}\text { Ghich is not always available. } \\
\text { whic is determine, }\end{array}$ \\
\hline
\end{tabular}

\section{TTDD}

Two-Tier Data Dissemination (TTDD) proposed Luo et al. TTDD is helps to solve multiple mobile sink problem. TTDD uses sensor nodes at grid points for data transmission. Sink does not send data to the send data queries it builds grid structure with sensors closest to grid points.

Table 8. Merit and Limitation of TTDD

\begin{tabular}{|c|c|}
\hline Merit & Limitation \\
\hline $\begin{array}{l}\text { Resolves multiple-mobile sinks and sink-moving } \\
\text { problems in large-scale. } \\
\text { Suited to event-detecting WSNs among irregular data } \\
\text { traffic. }\end{array}$ & $\begin{array}{l}\text { Latency is large. } \\
\text { Low energy efficiency. } \\
\text { Sensor nodes are need to be stationary and location- } \\
\text { aware. }\end{array}$ \\
\hline
\end{tabular}




\section{SLGC}

SLGC clustering algorithm was proposed by Delavar et al. This algorithm organizes the network into grids in each cluster, cluster head sends the data thus the network lifetime increases and the consumption of energy is reduces.

Table 9. Merit and Limitation of SLGC

\begin{tabular}{|l|l|}
\hline Merit & Limitation \\
\hline Energy consumption is lower compared to LEACH. & Due to large data communication large overhead occur. \\
\hline
\end{tabular}

\section{CHAIN CLUSTER BASED ROUTING ALGORITHM}

\section{PEGASIS}

Power-Efficient Gathering in Sensor Information System is proposed by Lindey et al. Each node communicate with its closest neighbours and become the leader for transmission to sink, one by one.

In PEGASIS, the nodes are gather in the form of chain themselves, they first get locations of all nodes and form chain using greedy algorithm.

Table. 11 Merit and Limitation of PEGASIS

\begin{tabular}{|l|l|}
\hline Merit & Limitations \\
\hline $\begin{array}{l}\text { Reduces overhead due to dynamic cluster formation. } \\
\begin{array}{l}\text { Decreases number of data transmission due to chain } \\
\text { of data aggregation. }\end{array}\end{array}$ & $\begin{array}{l}\text { Not suitable for networks with time-varying topologies. } \\
\text { Energy load is distributed uniformly in network. } \\
\text { become bottleneck. }\end{array}$ \\
$\begin{array}{l}\text { Network is not scalable, due to global knowledge of } \\
\text { network. }\end{array}$ \\
\hline
\end{tabular}

\section{CCS}

Concentric Clustering Scheme (CCS) proposed by Jung et. al. In CCS base station location is consider to increase network lifetime to enhance performance.

CCS network is divided into concentric circular tracks (Level), which indicates different cluster. Track close to the base station assigned level 1, similarly level assigned according to the distance increases between the base stations and track. On each level, nodes form chain inside track, one node is selected as cluster head.

In each level, all nodes transmit their data to nearest node along chain. Nodes which received data fuses its own data and transmit to the next node. Cluster head of each level transmits data to lesser cluster head. Then level 1, cluster head transmit all the data to base station.

Table 12. Merit and Limitation of CCS

\begin{tabular}{|l|l|}
\hline Merit & Limitations \\
\hline $\begin{array}{l}\text { Energy consumption is reduced due to lesser distance } \\
\text { between base station and cluster head. }\end{array}$ & $\begin{array}{l}\text { Unbalance node distribution at each level, levels with } \\
\text { less number of node deplete their energy first. }\end{array}$ \\
$\begin{array}{l}\text { Reduced data flow from BS by dividing network in } \\
\text { concentric clusters. }\end{array}$ & Long chain node communication causes large delays. \\
\hline
\end{tabular}

\section{TSC}

Track-Sector Clustering (TSC) proposed by Gautam et al.The network get partition into concentric circular tracks and triangular sectors. This division of tracks and sectors saves energy because it minimizes redundant data transmission and create shortest distance between cluster head and base station[13]. The cluster formation in TSC takes place in an area under curved form by intersection of circular track and triangular sector.

The execution process can be divided into four phases:

Track setup: Base station set each node in particular concentric circular track at the geometric centre. Total number of tracks depends on the node density and location of base station.

Sector setup and Cluster head Selection: Base station construct the sectors and select their cluster head.

Chain Construction: By intersection of tracks and sectors chains are formed within each cluster.

Data transmission: In the cluster all the member of the nodes receive and transmit data to neighbouring node.

Finally data is transmitted to the base station by multiple hops. 


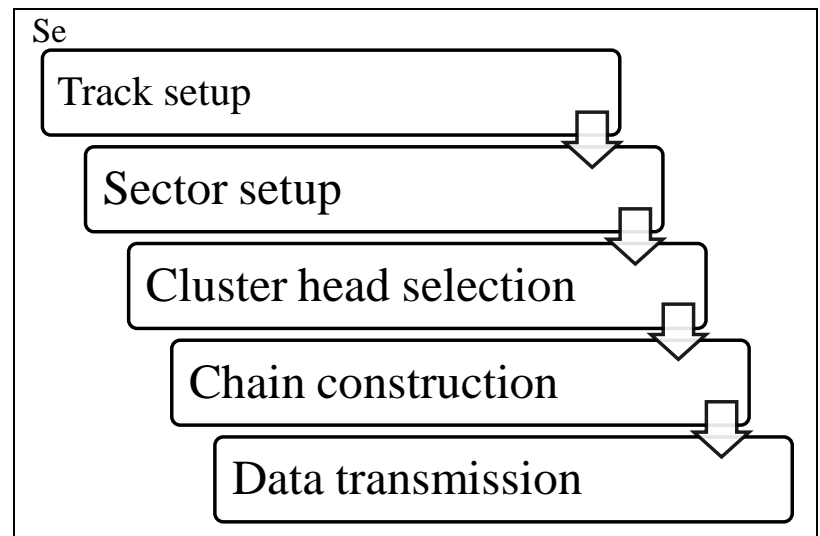

Fig.5. Execution process of TSC

Table 13. Merit and Limitation of TSC

\begin{tabular}{|l|l|}
\hline Merit & Limitations \\
\hline $\begin{array}{l}\text { Reduces redundant data transmission by breaking } \\
\text { long chain into smaller chain }\end{array}$ & $\begin{array}{l}\text { Unbalance energy consumption as level of small number } \\
\text { of nodes deplete their energy first. }\end{array}$ \\
\hline
\end{tabular}

\section{CONTROVERSY IN CLUSTER BASED ROUTING}

The countless research has been done to question the failing of clustering technique to promote the originality of cluster based routing methods but still several issues to be addressed for efficient use of cluster based routing techniques. Some open issues need to be addressed are:

Calculation and Selection of cluster heads(CHs): $\mathrm{CH}$ is utilize in computational and communication energy model for clustering schemes. The availability of adjacent cluster head and cluster head role rotation in neighbouring cluster is importantin aggregation of data clusters.

Topology Changes of Network: Topology changes owned in realization of WSNs, so it focus on the strength of clustering methods.

Network Expedition: It is desire to expand the monitoring area with sensor nodes. It became necessary to check the adaptability and scalability of newly added nodes with different resource capability of clustering schemes.

Transient Fault Management: Temporary link failure needs more investigation due to fault management Surplus Nodes:Redundant number of nodes are minimize

\section{OPEN RESEARCH AREAS IN CLUSTER-BASED WSNS}

Cluster-based schemes recently proposed lot of critical concern for the deployment of wireless sensor network[17]. Still there are some latest research issues in cluster-based WSNs are as follows:

Cluster Head Selection: It is based on the parameters such as number of $\mathrm{CHs}$, round number, time interval, node ID, and location information.

The issues it faces are as follows

$>$ Sheer probabilistic.

$>$ Network resources and parameters are not consider.

$>$ Sensor nodes have to periodically update latest information.

$>$ Imposes extra overhead to heavy-loaded CHs.

Cluster Formation: It is based event location and direction of data flow also minimizes the energy expenditure in clustering.

The issues it faces are as follows

$>$ Extra overhead in terms of communication and process on the regions that does not have event to report.

Nodes have to periodically transmit their sensor reading to BS.

Data aggregation: Based on the optimum selection of aggregation efficiency, optimal cluster radius to achieve most efficient data aggregation.

The issues it faces are as follows:

$>$ It increases the memory storage and delay time. 
$>$ Degrade fault detection mechanism of network.

$>$ Causes spatial and temporal schemes issues.

Data Communication: It is based on the long distance communication of CHs and BS, uses CDMA slots to avoid radio interference. Select optimum number of relay nodes.

The issues it faces are as follows:

Extra overload to heavy loaded $\mathrm{CHs}$ that depletes the closeness of $\mathrm{CHs}$ to BS.

$>$ Communication between $\mathrm{CH}-\mathrm{CH}$ might be long distance transmission for large scale networks.

\section{CONCLUSION}

The existing routing strategies in wireless sensor network and its corresponding routing protocols had been explained. Clustering based routing algorithm is best technique to reduce energy consumption and also provide stability and scalability in the network. We discuss the advantage and taxonomy of cluster-based routing algorithms in WSN. This paper will very useful for research group those are interested in development, modification or optimization of routing algorithms for WSNs.

\section{REFERENCES}

[1] F. Akyildiz,W. Su, Y. Sankarasubramaniam, and E. Cayirci, “A Survey on Sensor Netowrks,” IEEE Communications Magazine, vol. 40, no. 8,pp. 102-114, Aug 2002.

[2] Al-Karaki, J.N.; Kamal, A.E. Routing techniques in wireless sensor networks: A survey. IEEE Wireless Communication. $2004,11,6-28$.

[3] Ding, P.; Holliday, J.; Celik, A. Distributed Energy Efficient Hierarchical Clustering for Wireless Sensor Networks. In Proceedings of the 8th IEEE International Conference on Distributed Computing in Sensor Systems (DCOSS), Marina Del Rey, CA, USA, 8-10 June 2005; pp. 322339.

[4] Yick, J., Biswanath, M., Ghosal, D., "Wireless Sensor Network Survey” Computer Networks, vol.52(12):2292-2330,2008

[5] W.B Heinzelman, A.P.Chandrakasan, H.Balakrishnan,"Application specific protocol architecuture for wireless micro sensor April 2003.

[6] S. Bandyopadhyay and E.J Coyle," An Energy Efficient Hierarchical Clustering Alogrithm for Wireless Sensor Networks",IEEE INFOCOM, April 2003.

[7] S. Soro, W. B Heinzelman, "Prolonging the Lifetime of Wireless Sensor Network via Unequal Clustering", in proceeding of 19 $9^{\text {th }}$ IEEE International Performance, Computing and Communication Conference, pp .535-540, 2005.

[8] X. Liu and J. Shi, "Clustering Routing Algorithm In Wireless Sensor Network": KSII Transactions on Internet and Information Systems, vol.6, no.7,pp. 1735-1755, 2012.

[9] F. Awad,'Energy-Efficient and Converge-Aware Clustering in Wireless Sensor Networks," Wireless Engineering and Technology, vol.03, no. 03, pp. 142-151,2012.

[10] Anno, J., Barolli, L.; A Xhafa, F., ; Koyama, A. A Cluster Head Decision System for Sensor Network Using Fuzzy Logic and Number of Neighbour Node. In Proceedings of the $1^{\text {st }}$ IEEE International Conference on Ubi-Media Computing, Gansu, China,14-20 July 2008;pp . 50-56.

[11] Ahmed A. Ahmed, Hongchi Shi, and Yi Shang." A SURVERY ON NETWORK PROTOCOLS FOR WIRELESS SENSOR NETWORKS" 07803-7724-9/03/\$17. 00 IEEE 2003.

[12] Ameer Ahmed Abbasi, Mohamed Younis.” A Survey on Clustering Algorithm for Wireless Sensor Networks"(IEEE) 0140-3664/\$ 2007.

[13] Amandeep Kaur Mann, Navneet Kaur "SURVEY PAPER ON CLUSTERING TECHNIQUES", ISSN: 2278 - 7798 International Journal of Science, Engineering and Technology Research (IJSETR) Volume 2, Issue 4, April 2013.

[14] Rui Xu, Donald C. Wunsch,"Survey Of Clustering Algorithms”.IEEE TRANSACTIONS ON NEURAL NETWORKS, VOL. 16, NO. 3, MAY 2005.

[15] Congfeng Jiang,Daomin Yuan,Yinghui Zhao,'Towards Clustering Algorithms in Wireless Sensor Networks-A Survey".978-1-4244-29486/09/\$25.00 @2009 IEEE.

[16] Santar Pal Singh, S.C. Sharma, "A Survey on Cluster Based Routing Protocols in Wireless Sensor Sensor Networks". International Conference on Advanced Computing Technologies and Applications 2015

[17] Salim EL Khediri, Nejah Nasri, Anne Wei, Abdennaceur Kachouri,” A New Approach for Clustering in Wireless Sensors Networks Based on LEACH."1877-0509 @ 2014.

[18] M. Aslam, N. Javaid, A. Rahim, U. Nazir, A. Bibi, Z. A. Khan,” Survey of Extended LEACH-Based Clustering Routing Protocols for Wireless Sensor Networks". arXiv1207.2609 v1 2012.

[19] Vivek Katiyar, Narottam Chand, Surender Soni.” Clustering Algorithms for Heterogeneous Wireless Sensor Network: A Survey" ISSN - 0976-4259(IJAER).

[20] AsimZeb,A.K.M.MuzahidulIslam, Mahdi Zareei, IshtiakAlMamoon, Nafees Mansoor, SabariahBaharun, Yoshiaki Katayama, and Shozo Komaki."Clustering Analysis in Wireless Sensor Networks: The Ambit of Performance Metrics and Schemes Taxonomy"(IJDSN), 2016 